\title{
Pulmonary exacerbation in adults with bronchiectasis: a consensus definition for clinical research
}

\author{
Adam T. Hill ${ }^{1,26}$, Charles S. Haworth ${ }^{2,26}$, Stefano Aliberti (10 ${ }^{3}$, Alan Barker ${ }^{4}$, \\ Francesco Blasi ${ }^{3}$, Wim Boersma ${ }^{5}$, James D. Chalmers ${ }^{6}$, Anthony De Soyza ${ }^{7}$, \\ Katerina Dimakou ${ }^{8}$, J. Stuart Elborn ${ }^{9}$, Charles Feldman ${ }^{10}$, Patrick Flume ${ }^{11}$, \\ Pieter C. Goeminne ${ }^{12,13}$, Michael R. Loebinger ${ }^{14}$, Rosario Menendez ${ }^{15}$, \\ Lucy Morgan ${ }^{16}$, Marlene Murris ${ }^{17}$, Eva Polverino ${ }^{18}$, Alexandra Quittner ${ }^{19}$, \\ Felix C. Ringshausen ${ }^{20}$, Gregory Tino ${ }^{21}$, Antoni Torres ${ }^{18}$, Montserrat Vendrell ${ }^{22}$, \\ Tobias Welte ${ }^{20}$, Rob Wilson ${ }^{14}$, Conroy Wong ${ }^{23}$, Anne $0^{\prime}$ Donnell ${ }^{24,27}$ and \\ Timothy Aksamit ${ }^{25,27}$ for the EMBARC/BRR definitions working group
}

@ERSpublications

An expert conference has developed a consensus definition of a bronchiectasis exacerbation for clinical research http://ow.ly/oKPY309yTaX

Cite this article as: Hill AT, Haworth CS, Aliberti S, et al. Pulmonary exacerbation in adults with bronchiectasis: a consensus definition for clinical research. Eur Respir J 2017; 49: 1700051 [https://doi.org/ 10.1183/13993003.00051-2017].

ABSTRACT There is a need for a clear definition of exacerbations used in clinical trials in patients with bronchiectasis. An expert conference was convened to develop a consensus definition of an exacerbation for use in clinical research.

A systematic review of exacerbation definitions used in clinical trials from January 2000 until December 2015 and involving adults with bronchiectasis was conducted. A Delphi process followed by a round-table meeting involving bronchiectasis experts was organised to reach a consensus definition. These experts came from Europe (representing the European Multicentre Bronchiectasis Research Collaboration), North America (representing the US Bronchiectasis Research Registry/COPD Foundation), Australasia and South Africa.

The definition was unanimously approved by the working group as: a person with bronchiectasis with a deterioration in three or more of the following key symptoms for at least $48 \mathrm{~h}$ : cough; sputum volume and/or consistency; sputum purulence; breathlessness and/or exercise tolerance; fatigue and/or malaise; haemoptysis AND a clinician determines that a change in bronchiectasis treatment is required.

The working group proposes the use of this consensus-based definition for bronchiectasis exacerbation in future clinical research involving adults with bronchiectasis. 
Affiliations: ${ }^{1}$ Royal Infirmary and University of Edinburgh, Edinburgh, UK. ${ }^{2}$ Cambridge Centre for Lung Infection, Papworth Hospital, Cambridge, UK. ${ }^{3}$ Respiratory Unit, IRCCS Fondazione Ca' Granda Ospedale Maggiore Policlinico, Dept of Pathophysiology and Transplantation, University of Milan, Milan, Italy. ${ }^{4}$ Dept of Pulmonary and Critical Care Medicine, Oregon Health and Science University, Portland, OR, USA. ${ }^{5}$ Dept of Pulmonary Diseases, Medical Centre Alkmaar, Alkmaar, The Netherlands. ${ }^{6}$ College of Medicine University of Dundee, Ninewells Hospital and Medical School, Dundee, UK. ${ }^{7}$ Institute of Cellular Medicine, Newcastle University and Bronchiectasis Service Freeman Hospital, Newcastle upon Tyne, UK. ${ }^{8} 5$ th Pulmonary Dept, "Sotiria" Chest Hospital, Athens, Greece. 'Imperial College and Royal Brompton Hospital, London, UK. ${ }^{10}$ Charlotte Maxeke Johannesburg Academic Hospital and Faculty of Health Sciences, University of the Witwatersrand, Johannesburg, South Africa. ${ }^{11}$ Dept of Medicine and Pediatrics, Medical University of South Carolina, Charleston, SC, USA. ${ }^{12}$ Dept of Respiratory Medicine, AZ Nikolaas, Sint-Niklaas, Belgium. ${ }^{13}$ Dept of Respiratory Disease, UZ Leuven, Leuven, Belgium. ${ }^{14}$ Host Defence Unit, Royal Brompton Hospital, London, UK. ${ }^{15}$ Pneumology Service, Universitary and Polytechnic Hospital La Fe, Valencia, Spain. ${ }^{16}$ Concord Hospital, Concord, Australia. ${ }^{17}$ Service de Pneumologie, Hopital Larrey, CHU de Toulouse, Toulouse, France. ${ }^{18}$ Fundaciò Clìnic, IDIBAPS, CIBERES, Hospital Clinic de Barcelona, Barcelona, Spain. ${ }^{19}$ Behavioral Health Systems Research, Miami, FL, USA. ${ }^{20}$ Dept of Respiratory Medicine, Hannover Medical School, Hannover, Germany.

${ }^{21}$ Dept of Medicine, Perelman School of Medicine at the University of Pennsylvania, Philadelphia, PA, USA.

${ }^{22}$ Bronchiectasis Group, IDIBGI, CIBERES, Dr Trueta University Hospital, Girona, Spain. ${ }^{23}$ Dept of Respiratory Medicine, Middlemore Hospital, Counties Manukau District Health Board, Auckland, New Zealand. ${ }^{24}$ Division of Pulmonary, Critical Care and Sleep Medicine, Georgetown University Medical Center, Washington, DC, USA.

${ }^{25}$ Mayo Clinic College of Medicine, Rochester, MN, USA. ${ }^{26}$ Joint first authors. ${ }^{27}$ Joint last authors.

Correspondence: Adam T. Hill, Dept of Respiratory Medicine, Royal Infirmary of Edinburgh, 51 Little France Crescent, Edinburgh, EH16 4SA, UK. E-mail: adam.hill318anhs.net

\section{Introduction}

Patients with bronchiectasis have frequent exacerbations that are a cause of significant morbidity and sometimes mortality, and are desirable to prevent [1]. The British Thoracic Society has defined exacerbations requiring antibiotics based on expert consensus as a deterioration in local symptoms (cough, increased sputum volume or change of viscosity, increased sputum purulence with or without increasing wheeze, dyspnoea, haemoptysis) and/or systemic upset [1].

Exacerbation frequency or time to next exacerbation are key end-points in clinical trials evaluating new treatments in adults with bronchiectasis. This is a view supported by investigators, clinicians involved in the care of bronchiectasis patients and individuals with bronchiectasis, and aligns with the current European Medicines Agency and US Food and Drug Administration mandates to use time to first exacerbation or the mean or median number of exacerbations in recent clinical trials of inhaled antibiotics [2,3]. However, heterogeneity in the definition of exacerbation used can hinder comparisons of treatment effect.

An international group of investigators with interest in bronchiectasis gathered at the first World Bronchiectasis Conference in Hannover, Germany (July 7, 2016) to address the definition of bronchiectasis exacerbation. The aim of this working group was to develop a consensus definition of exacerbation for clinical trials involving patients with bronchiectasis. This consensus definition is for clinically significant bronchiectasis in adults, i.e. persistent symptoms consistent with bronchiectasis plus confirmation of bronchiectasis with computed tomography scan of the chest, and excludes patients with cystic fibrosis (CF).

\section{Methods}

A systematic review of exacerbation definitions used in clinical research from January 2000 until December 2015, involving adults with bronchiectasis not related to CF, was conducted [2, 4-23]. Search terms used from a PubMed search were "definition" and "exacerbation" and "exacerbation definition". Studies related to review articles, descriptive cohorts and editorials were excluded.

Definitions of exacerbations were identified and individual criteria extracted. All identified criteria were then included in a Delphi process [24] involving bronchiectasis experts from Europe (representing the European Multicentre Bronchiectasis Research Collaboration (EMBARC)), North America (representing the US Bronchiectasis Research Registry/COPD Foundation (BRR)), Australasia and South Africa. Between May and June 2016, an online survey was conducted to determine which criteria, signs and symptoms identified by the systematic search should be included in a consensus definition. During the first round of the Delphi process, experts were able to add criteria that they deemed important. These new criteria were then graded in the further rounds.

There were three Delphi rounds to reach consensus. Criteria were graded as: 1, should not be a part of the exacerbation definition; 2 , possibly suggestive of an exacerbation; 3 , highly suggestive of an exacerbation; 4 , must be present to define an exacerbation. Criteria were excluded if $\geqslant 80 \%$ of participants rated them 1 or 2 and factors were considered for inclusion if $\geqslant 80 \%$ of participants rated them 3 or 4 , or the average score was $\geqslant 2$ on all three Delphi rounds. 
Shortlisted criteria were then discussed by the committee with majority voting used to determine the final criteria and structure of the definition.

\section{Results}

The systematic review identified 50 articles with 20 different definitions of exacerbation identified. More than $80 \%$ of published criteria included a requirement for antibiotic use, and the symptoms of increased dyspnoea, increased cough, increased sputum volume and a change in sputum colour. All other criteria were used in $<80 \%$ of definitions.

All possible criteria were considered in the Delphi survey. After three rounds, the Delphi process resulted in the following signs and symptoms scoring mean $\geqslant 2$ in the grading process (graded lowest to highest): haemoptysis, chest pain, decreased exercise tolerance, wheeze, hypoxia, fever, auscultation findings, patient self-reporting an exacerbation, systemic disturbance [13], fatigue, sputum viscosity, worsening shortness of breath, symptoms $\geqslant 24 \mathrm{~h}$, worsening sputum colour, increased cough, worsening sputum volume, symptoms $\geqslant 48 \mathrm{~h}$, exclusion of other causes and antibiotics used for the exacerbation (figure 1 ).

Although some previous criteria had used a combination of "major and minor" symptoms, the working group agreed ( $90 \%$ consensus) criterion based on a combination of symptoms with equal weighting was more operational.

Not all definitions identified in the systematic review required treatment with antibiotics or a change in treatment. Notably, in chronic obstructive pulmonary disease studies the concept of mild exacerbations that do not receive treatment is well established. In the case of bronchiectasis, $90 \%$ of our committee agreed that antibiotic treatment was not required to define an exacerbation, but $81 \%$ agreed that a change in management was required (which may include a new treatment with antibiotics and/or systemic steroids, or an increase in airways clearance, inhaled therapy or another intervention).

The outputs from the systematic review and subsequent Delphi process were discussed by the working group and a consensus ( $>80 \%$ participant approval) definition for a bronchiectasis exacerbation was agreed as: a person with bronchiectasis with a deterioration in three or more of the following key symptoms for at least $48 \mathrm{~h}$ : cough; sputum volume and/or consistency; sputum purulence; breathlessness and/or exercise

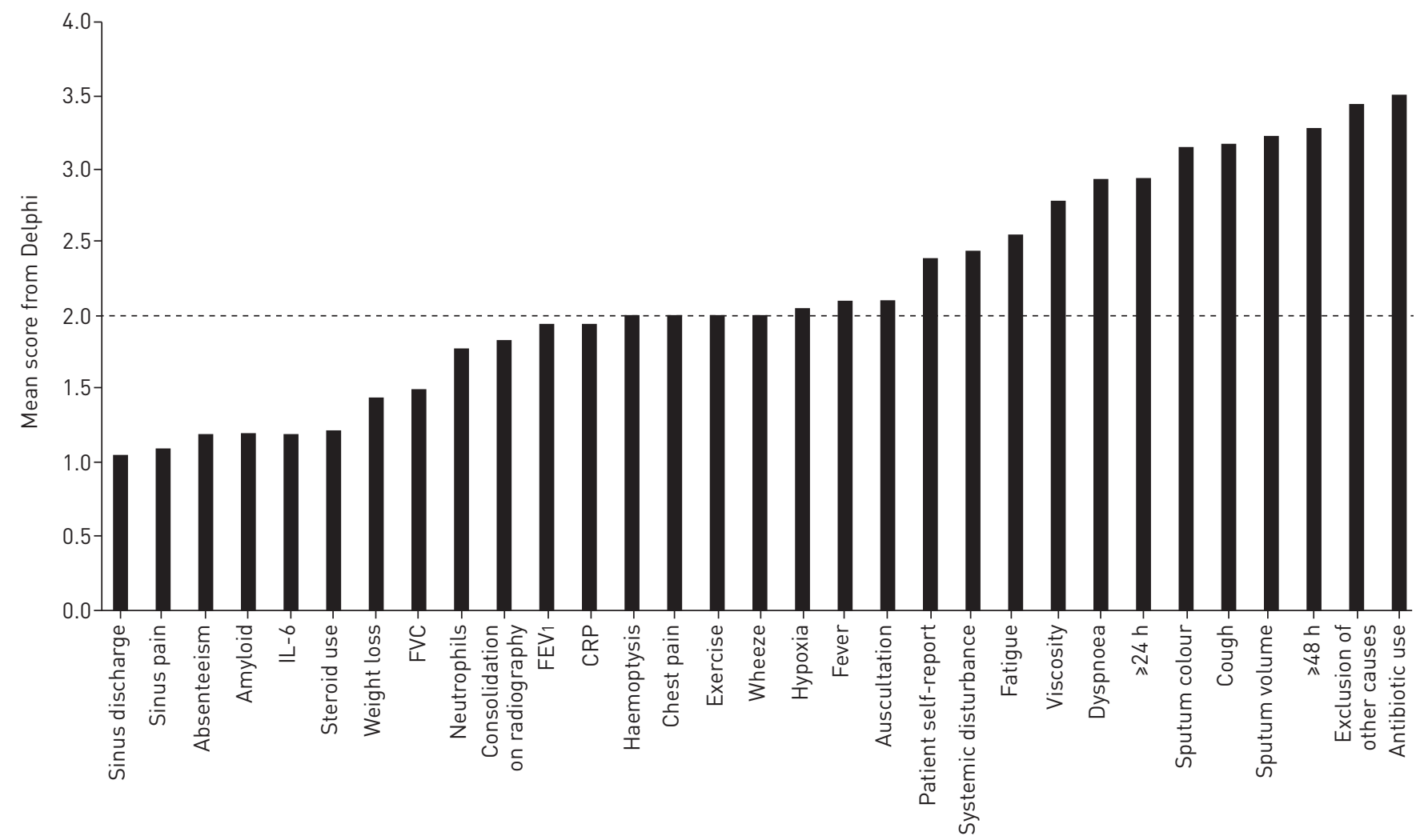

FIGURE 1 Results from the Delphi process. IL: interleukin; FVC: forced vital capacity; FEV1: forced expiratory volume in 1 s; CRP: C-reactive protein. The scores were graded as: 1 , should not be a part of the exacerbation definition; 2 , possibly suggestive of an exacerbation; 3 , highly suggestive of an exacerbation; 4 , must be present to define an exacerbation. Scores $\geqslant 2$ were targeted for discussion at the working group. 
tolerance; fatigue and/or malaise; haemoptysis AND a clinician determines that a change in bronchiectasis treatment is required (figure 2). This definition was unanimously approved by the working group.

The following factors, signs and symptoms did not reach a sufficient threshold for consideration to be included by the working group as part of the consensus definition: sinus discharge, sinus pain, absenteeism, systemic inflammatory markers such as neutrophil count, C-reactive protein (CRP), interleukin (IL)-6 and amyloid, steroid use, weight loss, reduction in forced expiratory volume in $1 \mathrm{~s}$ (FEV1) and/or forced vital capacity (FVC), and chest radiographic findings of pneumonia.

\section{Discussion}

This consensus definition of exacerbation may serve to standardise a key outcome measure of clinical trials involving adults with bronchiectasis and allow more meaningful comparisons of treatment effect between interventions. Although the remit of the working group was to define pulmonary exacerbation in adults with bronchiectasis in the context of prospective clinical trials, it is anticipated that this definition will inform patients and clinicians to more accurately identify exacerbations. We acknowledge that this definition is not a validated instrument, which might be more useful in determining if treatment of an exacerbation is efficacious. However, it does provide an agreed-upon definition by a group of bronchiectasis experts using a systematic approach intended to define a clinical end-point in the treatment of bronchiectasis and represents a proposed definition to be used for validation in future investigations.

The systematic review performed prior to the working group meeting provided an evidence-based platform for the factors included in the Delphi process. The subsequent outputs then enabled investigators to discuss and establish arguments so as to agree on the key components of the definition.

The consensus definition has three core components: collection of symptoms, time and decision to change treatment. Owing to the lack of evidence and validation data, any recommended criteria are preliminary and may be modified as more data become available.

Previous definitions used in trials had included a wide variety of symptoms but only six made the final list for this proposed definition. For some criteria it was deemed they were not related to exacerbation of lower airways disease (e.g. sinus disease and heart failure), while for others the criteria were felt to be infrequently measured in clinical practice to be relevant (e.g. CRP, serum IL-6 and serum amyloid) [1, 25, 26]. Spirometric measures were discounted because, unlike in CF, changes in FEV1 and FVC are not reliable indicators of exacerbation or response to treatment in patients with bronchiectasis [25]. Auscultatory findings were also felt to not be sufficiently bronchiectasis specific and are largely dependent on the severity of the predominant underlying lung condition. Chest radiograph changes were excluded as it can be difficult to distinguish pneumonia from mucus plugging, a finding that is common in bronchiectasis, and because most exacerbations occur in outpatient settings without access to radiology. A further important consideration during definition development was the lack of availability of some factors in domiciliary and certain healthcare settings. A symptom-based definition was therefore deemed preferable.

There was considerable debate regarding the requirement of a minimum number of criteria within the definition. It is assumed that a greater number of criteria met would increase the specificity of the diagnosis, and a consensus view that three out of six criteria be met was based upon a desire to achieve sufficient balance between sensitivity and specificity for use in clinical trials. This does not suggest that a patient cannot have an exacerbation when meeting only two out of six criteria. Nor is it known that such

FIGURE 2 Results of the exacerbation definition. "\#: other potential causes of clinical deterioration have been discounted.
Definition of a bronchiectasis pulmonary exacerbation for clinical trials

A person with bronchiectasis with a deterioration in three or more of the following key symptoms for at least $48 \mathrm{~h}$ :

1) Cough

2) Sputum volume and/or consistency

3) Sputum purulence

4) Breathlessness and/or exercise tolerance

5) Fatigue and/or malaise

6) Haemoptysis

AND a clinician determines that a change in bronchiectasis treatment is required\# 
exacerbations are the types we wish to prevent and future trials validating the number of symptoms that best define a diagnosis of exacerbation are needed.

Time was included in the definition to address the recognised day-to-day variability of symptoms in people with bronchiectasis. The international group recognised some patients feel worse over a 24-h period and then spontaneously improve without the need for augmentation or change of their treatment. Symptom duration of $\geqslant 48 \mathrm{~h}$ to be certain there was a real change in the condition was considered more appropriate than a threshold of only $24 \mathrm{~h}$. This element of the definition was thought to reduce inappropriate antibiotic prescribing and support adherence to the principal of antibiotic stewardship. If, however, there is a severe attack within $48 \mathrm{~h}$ necessitating treatment, such patients would still qualify with the proposed definition as they will have symptoms lasting $\geqslant 48 \mathrm{~h}$, as the treatment takes time to improve symptoms.

Finally, there is the inclusion of a need for intervention. Although most exacerbations are treated with antibiotics, the committee felt that not all exacerbations require antibiotic therapy as they could be self-limiting (e.g. viral illness) or respond to alternate therapy (e.g. steroids for allergic bronchopulmonary aspergillosis). Studies of specific medications may elect to define the specific intervention. Symptoms of sufficient severity warranting a change in bronchiectasis treatment were also thought to represent a minimum threshold for definition of exacerbation for potential intervention and purposes of development of a definition for clinical intervention trials. Formal grading of the severity of exacerbation into mild, moderate and severe was not felt to be critical for the objective of this work.

It is important to capture all exacerbations in which a physician makes a decision to treat as part of the outcomes in clinical trials. The purpose of a definition is not to override the clinical judgement of physicians but to achieve some objective verification of that decision in multicentre, often international trials, where the threshold for that decision to treat is variable.

In conclusion, the working group comprising bronchiectasis experts from Europe (representing EMBARC), North America (representing BRR), Australasia and South Africa proposes the use of this consensus-based definition for bronchiectasis exacerbation (figure 2) in clinical trials involving adults with bronchiectasis. Although there was no representation from Asia, we believe this definition will be applicable internationally but will need to be validated in different settings. Although the evidence base was limited, international experts propose this workable definition and the plan is that there will be validation of this definition in future trials.

\section{Acknowledgement}

The authors thank the European Lung Foundation (ELF) and the ELF/EMBARC patient advisory group for providing patient input into the project.

\section{References}

1 Pasteur MC, Bilton D, Hill AT, et al. British Thoracic Society guideline for non-CF bronchiectasis. Thorax 2010; 65: Suppl. 1, i1-i58.

2 Serisier DJ, Bilton D, De Soyza A, et al. Inhaled, dual release liposomal ciprofloxacin in non-cystic fibrosis bronchiectasis (ORBIT-2): a randomised, double-blind, placebo-controlled trial. Thorax 2013; 68: 812-817.

3 Wilson R, Welte $\mathrm{T}$, Polverino $\mathrm{E}$, et al. Ciprofloxacin dry powder for inhalation in non-cystic fibrosis bronchiectasis: a phase II randomised study. Eur Respir J 2013; 41: 1107-1115.

4 Altenburg J, de Graaff CS, Stienstra Y, et al. Effect of azithromycin maintenance treatment on infectious exacerbations among patients with non-cystic fibrosis bronchiectasis: the BAT randomized controlled trial. JAMA 2013; 309: 1251-1259.

5 Bilton D, Daviskas E, Anderson SD, et al. Phase 3 randomized study of the efficacy and safety of inhaled dry powder mannitol for the symptomatic treatment of non-cystic fibrosis bronchiectasis. Chest 2013; 144: 215-225.

6 Bilton D, Tino G, Barker AF, et al. Inhaled mannitol for non-cystic fibrosis bronchiectasis: a randomised, controlled trial. Thorax 2014; 69: 1073-1079.

7 Chalmers JD, Smith MP, McHugh BJ, et al. Short- and long-term antibiotic treatment reduces airway and systemic inflammation in non-cystic fibrosis bronchiectasis. Am J Respir Crit Care Med 2012; 186: 657-665.

8 Kellett F, Robert NM. Nebulised 7\% hypertonic saline improves lung function and quality of life in bronchiectasis. Respir Med 2011; 105: 1831-1835.

9 Mandal P, Chalmers JD, Graham C, et al. Atorvastatin as a stable treatment in bronchiectasis: a randomised controlled trial. Lancet Respir Med 2014; 2: 455-463.

10 Hernando R, Drobnic ME, Cruz MJ, et al. Budesonide efficacy and safety in patients with bronchiectasis not due to cystic fibrosis. Int J Clin Pharm 2012; 34: 644-650.

11 Lee AL, Hill CJ, Cecins N, et al. The short and long term effects of exercise training in non-cystic fibrosis bronchiectasis - a randomised controlled trial. Respir Res 2014; 15: 44.

12 Barker AF, O'Donnell AE, Flume P, et al. Aztreonam for inhalation solution in patients with non-cystic fibrosis bronchiectasis (AIR-BX1 and AIR-BX2): two randomised double-blind, placebo-controlled phase 3 trials. Lancet Respir Med 2014; 2: 738-749.

13 Martínez-García MA, Soler-Cataluña JJ, Catalán-Serra P, et al. Clinical efficacy and safety of budesonideformoterol in non-cystic fibrosis bronchiectasis. Chest 2012; 141: 461-468.

14 Murray MP, Govan JR, Doherty CJ, et al. A randomized controlled trial of nebulized gentamicin in non-cystic fibrosis bronchiectasis. Am J Respir Crit Care Med 2011; 183: 491-499. 
15 Murray MP, Pentland JL, Hill AT. A randomised crossover trial of chest physiotherapy in non-cystic fibrosis bronchiectasis. Eur Respir J 2009; 34: 1086-1092.

16 Nicolson CH, Stirling RG, Borg BM, et al. The long term effect of inhaled hypertonic saline 6\% in non-cystic fibrosis bronchiectasis. Respir Med 2012; 106: 661-667.

17 Patterson JE, Hewitt O, Kent L, et al. Acapella versus 'usual airway clearance' during acute exacerbation in bronchiectasis: a randomized crossover trial. Chron Respir Dis 2007; 4: 67-74.

18 Serisier DJ, Martin ML, McGuckin MA, et al. Effect of long-term, low-dose erythromycin on pulmonary exacerbations among patients with non-cystic fibrosis bronchiectasis: the BLESS randomized controlled trial. JAMA 2013; 309: 1260-1267.

19 Valery PC, Morris PS, Byrnes CA, et al. Long-term azithromycin for Indigenous children with non-cystic-fibrosis bronchiectasis or chronic suppurative lung disease (Bronchiectasis Intervention Study): a multicentre, double-blind, randomised controlled trial. Lancet Respir Med 2013; 1: 610-620.

20 Wong C, Jayaram L, Karalus N, et al. Azithromycin for prevention of exacerbations in non-cystic fibrosis bronchiectasis (EMBRACE): a randomised, double-blind, placebo-controlled trial. Lancet 2012; 380: 660-667.

21 Kapur N, Masters IB, Morris PS, et al. Defining pulmonary exacerbation in children with non-cystic fibrosis bronchiectasis. Pediatr Pulmonol 2012; 47: 68-75.

22 Haworth CS, Foweraker JE, Wilkinson P, et al. Inhaled colistin in patients with bronchiectasis and chronic Pseudomonas aeruginosa infection. Am J Respir Crit Care Med 2014; 189: 975-982.

23 Bilton D, Henig N, Morrissey B, et al. Addition of inhaled tobramycin to ciprofloxacin for acute exacerbations of Pseudomonas aeruginosa infection in adult bronchiectasis. Chest 2006; 130: 1503-1510.

24 Murphy MK, Black NA, Lamping DL, et al. Consensus development methods, and their use in clinical guideline development. Health Technol Assess 1998; 2: 1-88.

25 Murray MP, Turnbull K, Macquarrie S, et al. Assessing response to treatment of exacerbations of bronchiectasis in adults. Eur Respir J 2009; 33: 312-318.

26 Vendrell M, de Gracia J, Olveira C, et al. Diagnostico y tratamiento de las bronquiectasias. SEPAR. [Diagnosis and treatment of bronchiectasis. Spanish Society of Pneumology and Thoracic Surgery.] Arch Bronconeumol 2008; 44: $629-640$. 